\title{
Response to Vemurafenib in Metastatic Triple-Negative Breast Cancer Harbouring a BRAF V600E Mutation: A Case Report and Electronically Captured Patient-Reported Outcome
}

\author{
Magdalena Pircher ${ }^{a} \quad$ Thomas Winder $^{b} \quad$ Andreas Trojan ${ }^{a, c}$ \\ aOnkozentrum Zürich, Zurich, Switzerland; bSwiss Tumor Molecular Institute, Zurich, \\ Switzerland; 'Swiss Tumor Institute, Zurich, Switzerland
}

\section{Keywords}

BRAF V600E · Metastatic triple-negative breast cancer - Vemurafenib · Electronically captured patient-reported outcomes · ePROs · Consilium smartphone app

\begin{abstract}
Effective treatment options are still scarce for metastatic triple-negative breast cancers. An increasing interest in the mutational landscape of this disease will facilitate novel therapeutic strategies in a variety of cancers. Here we report the case of a 38-year-old female patient who developed multiple lung metastasis of a triple-negative breast cancer 2 years after the completion of local therapy. When she progressed after two palliative chemotherapy lines and local electroporation, a next-generation sequencing revealed a BRAF V600E mutation for which we initiated therapy with the BRAF inhibitor vemurafenib. Radiological improvement was already evident after 3 months and has been ongoing for 19 months so far with very few side effects, as is demonstrated by electronically captured patient-reported outcomes. To our knowledge, this is the first published case where a BRAF V600E-mutated advanced triplenegative breast cancer was successfully treated with vemurafenib.
\end{abstract}

(C) 2021 The Author(s).

Published by S. Karger AG, Basel

\section{Introduction}

Triple-negative breast cancers (TNBCs) are characterized by the absence of oestrogen receptors, progesterone receptors, and Her2, and account for $15-20 \%$ of all breast cancers [1]. They represent the breast cancer subgroup with the worst prognosis, since they are typi- 
cally high-grade tumours, although low-grade tumours can rarely occur and are associated with a better prognosis [2].

Especially for the metastatic setting, beside classical chemotherapy and newly immunotherapy, effective treatment options are still scarce, leading to an increasing interest in the mutational landscape of TNBC in order to reveal new potential therapeutic targets. Only recently have genomic profiles of TNBC been defined using whole-exome sequencing $[1,2]$. To the best of our knowledge, no case has been published to date in which a metastatic TNBC harbouring a BRAF V600E mutation was treated with a small molecule targeting the BRAF kinase.

Vemurafenib is a low-molecular-weight molecule for the inhibition of the mutated serinethreonine kinase BRAF. It selectively binds to the ATP binding site of BRAF V600E kinase and inhibits its activity. Vemurafenib is especially used in the treatment of patients with nonresectable, advanced melanoma that harbour a BRAF V600E mutation. Furthermore, it has been tested in other cancer types in which a BRAF V600E mutation is present, namely papillary thyroid cancer, colorectal cancer, non-small-cell lung cancer, ovarian cancer, ErdheimChester disease, Langerhans cell histiocytosis, and hairy cell leukaemia [3-5].

Patient-reported outcomes (PROs), such as symptoms and functional status, are commonly measured in clinical trials. There is growing interest in integrating electronically captured PROs (ePROs) into routine clinical practice during chemotherapy and immunotherapeutic interventions [6]. The smartphone app Consilium is intended to continuously allow oncologists to monitor the progress of patients' symptoms, since ePROs create symptom progression charts based on structured patient entries and also notify a patient to contact the treatment centre in case symptoms are out of the acceptable range (Fig. 1) [7].

\section{Case Presentation}

We report the case of a 38-year-old white female accountant that was first diagnosed with node-positive TNBC at the age of 31 years after she had discovered a lump in her right breast. Her mother experienced breast cancer disease at the age of 50 years, but genetic testing of the patient discovered no BRCA1/BRCA2 mutation. The patient was a healthy nonsmoker with only social alcohol and moderate meat intake before diagnosis.

She underwent neoadjuvant chemotherapy with carboplatin, paclitaxel, and liposomal doxorubicin followed by mastectomy of the right breast with reconstruction and axillary lymphonodectomy. However, a histologic examination showed no pathological complete remission and we started an adjuvant chemotherapy with cyclophosphamide, methotrexate, and 5-fluorouracil (5-FU) once the adjuvant radiation therapy of the right chest wall and the lymphatic drainage pathways $(25 \times 1.8=45 \mathrm{~Gy})$ was completed. Methotrexate was stopped only after 1 month because of strong side effects (nausea, emesis) and we continued therapy with oral cyclophosphamide and oral 5-FU (capecitabine) for 3 months. However, 2 years after surgery, $x$-ray of the chest revealed an unclear lesion in the right lung. Beside this lesion (16 $\mathrm{mm}$ ), a positron emission tomography-computed tomography (CT) scan showed another small suspicious lesion in the lingula and unclear activity in the inguinal lymph nodes on the left side. Biopsy of the lesion in the right lung confirmed a metastasis of the known TNBC. We initiated first-line palliative chemotherapy with weekly carboplatin and paclitaxel, resulting in a partial remission in the CT scan after 3 months. The residual lung metastasis in the right lung was treated with irreversible electroporation. However, only 7 months later a local recurrence in the right breast was diagnosed and surgically resected. Palliative chemotherapy was restarted with weekly paclitaxel and carboplatin for another 3 months, but unfortunately a CT scan revealed progression of the lung metastasis in the right lung. A second-line chemo-

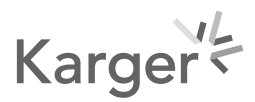




\section{Case Reports in Oncology}

\begin{tabular}{l|l}
\hline Case Rep Oncol 2021;14:616-621 \\
\hline DOI: 10.1159/000513905 & $\begin{array}{l}\text { ○ 2021 The Author(s). Published by S. Karger AG, Basel } \\
\text { www.karger.com/cro }\end{array}$ \\
\hline
\end{tabular}

Pircher et al.: Vemurafenib in BRAF-Mutated Metastatic Triple-Negative Breast Cancer
Fig. 1. History chart of ePROs as reported from the patient on her mobile device during treatment with vemurafenib. Blue, well-being; dark red, rash; light red, hand-foot syndrome; yellow, loss of appetite; olive green, nausea; orange, headache; green, cold symptoms; light purple, sensory disturbance; dark purple, gait disorder.

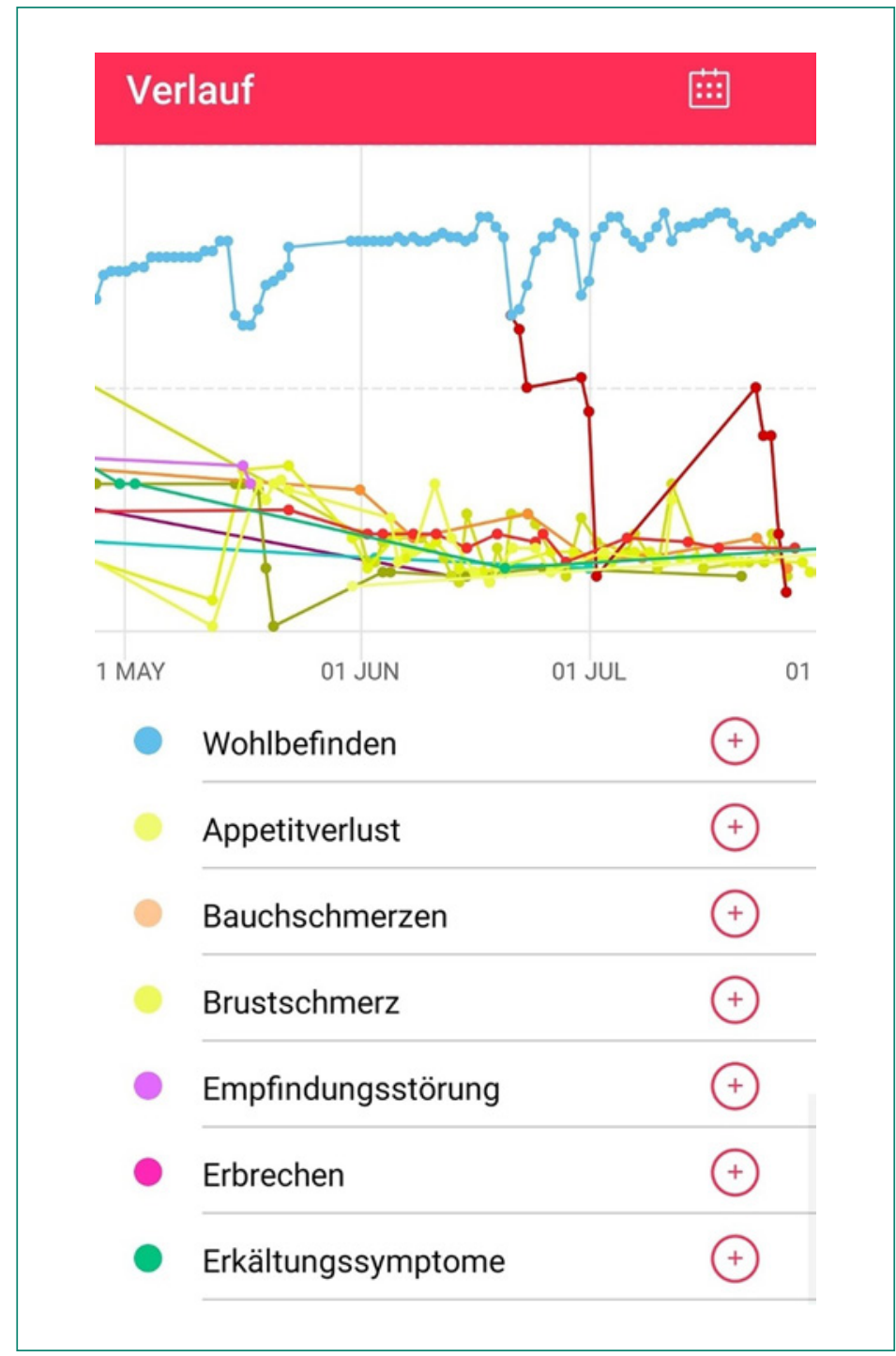

therapy was initiated with three-weekly liposomal doxorubicin and cyclophosphamide for 2 months, but had to be stopped after only three applications due to strong side effects (nausea, emesis, diarrhoea). Because of the young age of the patient, the excellent performance status, and lacking reasonable therapy alternatives, we carried out a lung biopsy in order to conduct molecular testing in the fresh tumour tissue. Next-generation sequencing using FoundationOne ${ }^{\circledR} \mathrm{CDx}$ showed a BRAF V600E mutation. This was considered to be of potential therapeutic use since it is classified as an oncogene and a tyrosine kinase inhibitor such as vemurafenib was a therapeutic option.

We discussed this option and the lack of clinical evidence data with the patient and she then started therapy with vemurafenib $720 \mathrm{mg}$ orally twice daily after giving her written consent in May 2019. At the same time the patient was using an electronic diary (Consilium Care $^{\mathrm{TM}}$ ) for shared reporting of ePROs (Fig. 1) [6-8]. Previously she had given informed consent to the observational study for ePRO monitoring (https://clinicaltrials.gov/ct2/ show/NCT03578731) that analyses the utility and reliability of self-reported electronic symptom monitoring [6, 7]. 


\section{Case Reports in Oncology}
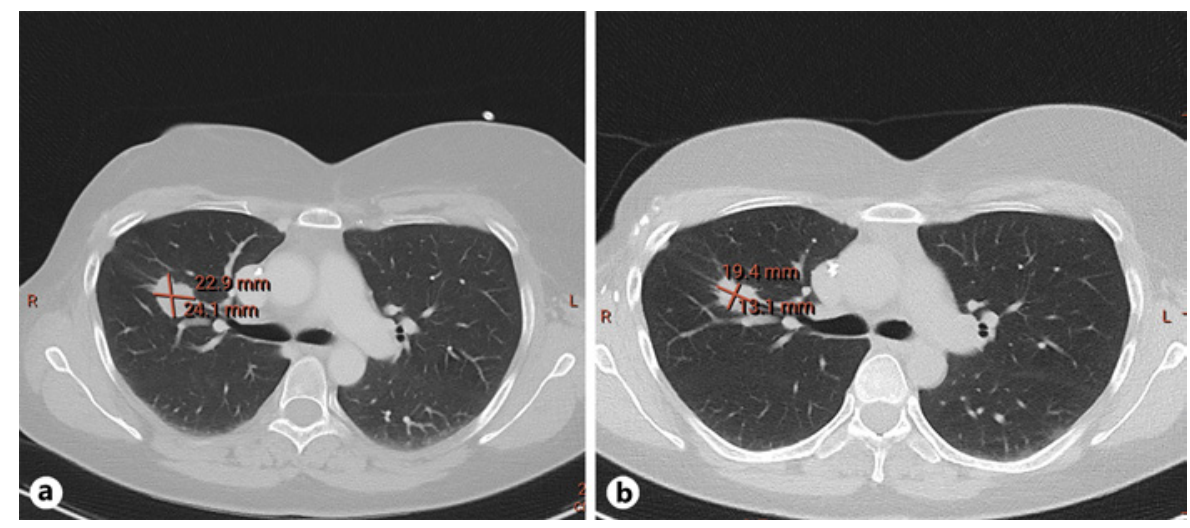

Fig. 2. CT scan of the lung showing the lung metastasis in the right lung measuring $24.1 \times 22.9 \mathrm{~mm}$ before vemurafenib treatment (a), and $19.4 \times 13.1 \mathrm{~mm} 3$ months after the initiation of vemurafenib treatment (b).

Because data regarding the use of vemurafenib in TNBC are missing, the patient's health insurance initially did not take care of the drug costs and the patient herself paid for the treatment at the beginning. The patient responded promptly, showing a partial remission of the lung metastasis in the CT scan performed 3 months after the initiation of treatment (Fig. 2a, b). Side effects were limited to slight nausea, rash, and hand-foot syndrome at the beginning of treatment. The lung metastasis has been radiologically stable up until now (19 months after therapy start) and the patient is still in a good clinical condition under ongoing treatment with vemurafenib.

\section{Discussion}

To the best of our knowledge, this is the first published case where a patient with a BRAF V600E-mutant advanced TNBC was successfully treated with vemurafenib. However, 1 case has been reported in the literature where a BRAF V600E-mutant metaplastic breast cancer patient with advanced disease showed an impressive but short response ( 8 weeks) to a combination therapy of the BRAF inhibitor dabrafenib and the MEK inhibitor trametinib, followed by a fulminant disease progression, leading to the patient's death only 12 weeks after therapy start [9]. In contrast, therapy with vemurafenib in our patient led to a rapid and to date persistent remission of an advanced TNBC harbouring a BRAF V600E mutation.

BRAF V600E mutations are rare in TNBC $(2-3 \%)[2,10]$, but they are of potential therapeutic interest because they can be targeted with kinase inhibitors such as vemurafenib. Beside BRAF, most somatic genetic alterations in TNBC occur in TP53 (80\%) [10], PTEN (35\%), RB 1 (30\%), and INPP4B (30\%) [10]. However, our patient showed none of the mutations mentioned above, and actually at present they cannot be successfully targeted therapeutically [1].

BRAF mutations are present in several other tumour types, including cutaneous melanomas (50\%), thyroid cancer (20-50\%), colorectal cancer (10\%), non-small-cell lung cancer (2-4\%), and hairy cell leukaemia (>90\%) [11]. They are crucial for the activation of the MAPK (RAS/RAF/MEK/ERK mitogen-activated protein kinase) signalling pathway, leading to a continuous stimulation of cell proliferation and inhibition of programmed cell death [3].

The efficacy of vemurafenib has been shown in multiple studies with melanoma patients that harbour a BRAF V600E mutation, reporting objective response rates in a range between

\section{Karger'}


50 and $80 \%[3,12]$. Regarding other tumour entities, in a basket study Hyman et al. [5] observed preliminary activity of vemurafenib in non-small-cell lung cancer, in ErdheimChester disease, and Langerhans cell histiocytosis. Furthermore, studies are ongoing in other cancer types that harbour a BRAF V600E mutation, including papillary thyroid cancer, colorectal cancer, ovarian cancer, and hairy cell leukaemia $[3,4]$.

Chemotherapy has been the mainstay of treatment for advanced TNBC for many years. Our patient was already pretreated with the most effective chemotherapeutic regimens in this setting (anthracyclines, taxanes, platin compounds). The remaining therapy alternatives reached objective response rates in the range of only $11-45 \%$ (i.e., eribulin mesylate, vinorelbine, ixabepilone, etoposide, gemcitabine) [13] and in our opinion presented no valuable alternative to vemurafenib with reported response rates between 50 and $80 \%$, as mentioned before [3]. Beside chemotherapy, new treatment options were recently launched with PARPis (polyadenosine-diphosphate-ribose polymerase inhibitors) for TNBC patients harbouring a BRCA mutation (BRCA mut) [14], and with immunotherapy additional to chemotherapy for TNBC patients with PD-L1 (programmed death-ligand 1)-positive tumours [15]. However, our patient showed neither a BRCA mutation nor positivity for PD-L1, and therefore did not classify for one of these therapies.

\section{Conclusion}

BRAF inhibition with vemurafenib has proven to be an effective therapy strategy in our patient with advanced TNBC harbouring a BRAF V600E mutation and is supported by moderate side effect profiling, as demonstrated by ePROs from the Consilium Care app. The potential for durable responses to vemurafenib supports the use of prospective molecular analysis of tumour tissue to screen patients with advanced TNBC for BRAF V600E mutations.

\section{Acknowledgements}

The authors thank Roche for providing the medication as part of an early access programme.

\section{Statement of Ethics}

Samples were obtained in accordance with the principles of the Declaration of Helsinki. Written informed consent was provided by the patient for publication of this case report and any accompanying images.

\section{Conflict of Interest Statement}

A.T. is an initiator and stock owner of mobile Health AG, a startup company that operates the Consilium smartphone app. He serves as a chief medical officer for the startup company.

\section{Karger's}




\section{Case Reports in Oncology}

\begin{tabular}{l|l}
\hline Case Rep Oncol 2021;14:616-621 \\
\hline DOI: 10.1159/000513905 & $\begin{array}{l}\text { ○ 2021 The Author(s). Published by S. Karger AG, Basel } \\
\text { www.karger.com/cro }\end{array}$ \\
\hline
\end{tabular}

Pircher et al.: Vemurafenib in BRAF-Mutated Metastatic Triple-Negative Breast Cancer

\section{Funding Sources}

This research was supported by the Swiss Tumour Institute Zurich, Switzerland, for conduct of a larger observational trial (ClinicalTrials.gov NCT03578731).

\section{Author Contributions}

A.T. followed the patient. T.W. performed the molecular advising. M.P. and A.T. wrote the paper. All of the authors reviewed and approved the final manuscript.

\section{Data Availability}

The datasets used and/or analysed during the current study are available from the corresponding author on reasonable request.

\section{References}

1 Garrido-Castro AC, Lin NU, Polyak K. Insights into molecular classifications of triple-negative breast cancer: improving patient selection for treatment. Cancer Discov. 2019;9(2):176-98.

2 Munzone E, Gray KP, Fumagalli C, Guerini-Rocco E, Láng I, Ruhstaller T, et al. Mutational analysis of triplenegative breast cancers within the International Breast Cancer Study Group (IBCSG) trial 22-00. Breast Cancer Res Treat. 2018;170(2):351-60.

3 Garbe C, Eigentler TK. Vemurafenib. Recent Results Cancer Res. 2018;211:77-89.

4 Kreitman RJ, Arons E. Update on hairy cell leukemia. Clin Adv Hematol Oncol. 2018;16(3):205-15.

5 Hyman DM, Puzanov I, Subbiah V, Faris JE, Chau I, Blay JY, et al. Vemurafenib in multiple nonmelanoma cancers with BRAF V600 mutations. N Engl J Med. 2015;373(8):726-36.

6 Egbring M, Far E, Roos M, Dietrich M, Brauchbar M, Kullak-Ublick GA, et al. A mobile app to stabilize daily functional activity of breast cancer patients in collaboration with the physician: a randomized controlled clinical trial. J Med Internet Res. 2016;18(9):e238.

7 Trojan A, Huber U, Brauchbar M, Petrausch U. Consilium smartphone app for real-world electronically captured patient-reported outcome monitoring in cancer patients undergoing anti-PD-L1-directed treatment. Case Rep Oncol. 2020 May-Aug;13(2):491-6.

8 Trojan A, Bättig B, Mannhart M, Seifert B, Brauchbar M, Egbring M. Collaborative review of ePROs for shared reporting in breast cancer patients does not affect the number of data entries. JMIR Cancer. Forthcoming. 2021.

9 Seo T, Noguchi E, Yoshida M, Mori T, Tanioka M, Sudo K, et al. Response to dabrafenib and trametinib of a patient with metaplastic breast carcinoma harboring a BRAF V600E mutation. Case Rep Oncol Med. 2020; 2020:2518383.

10 Shah SP, Roth A, Goya R, Oloumi A, Ha G, Zhao Y, et al. The clonal and mutational evolution spectrum of primary triple-negative breast cancers. Nature. 2012;486(7403):395-9.

11 Davies H, Bignell GR, Cox C, Stephens P, Edkins S, Clegg S, et al. Mutations of the BRAF gene in human cancer. Nature. 2002;417(6892):949-54.

12 Chapman PB, Hauschild A, Robert C, Haanen JB, Ascierto P, Larkin J, et al. Improved survival with vemurafenib in melanoma with BRAF V600E mutation. N Engl J Med. 2011;364(26):2507-16.

13 Zeichner SB, Terawaki H, Gogineni K. A review of systemic treatment in metastatic triple-negative breast cancer. Breast Cancer. 2016;10:25-36.

14 Robson M, Im SA, Senkus E, Xu B, Domchek SM, Masuda N, et al. Olaparib for metastatic breast cancer in patients with a germline BRCA mutation. N Engl J Med. 2017;377(6):523-33.

15 Schmid P, Adams S, Rugo HS, Schneeweiss A, Barrios CH, Iwata H, et al. Atezolizumab and nab-paclitaxel in advanced triple-negative breast cancer. N Engl J Med. 2018;379(22):2108-21. 